\title{
Fractional statistics and the butterfly effect
}

\author{
Yingfei Gu and Xiao-Liang Qi \\ Department of Physics, Stanford University, \\ Stanford, CA 94305, U.S.A. \\ E-mail: yfgu@stanford.edu, xlqi@stanford.edu
}

ABSTRACT: Fractional statistics and quantum chaos are both phenomena associated with the non-local storage of quantum information. In this article, we point out a connection between the butterfly effect in $(1+1)$-dimensional rational conformal field theories and fractional statistics in $(2+1)$-dimensional topologically ordered states. This connection comes from the characterization of the butterfly effect by the out-of-time-order-correlator proposed recently. We show that the late-time behavior of such correlators is determined by universal properties of the rational conformal field theory such as the modular S-matrix and conformal spins. Using the bulk-boundary correspondence between rational conformal field theories and $(2+1)$-dimensional topologically ordered states, we show that the late time behavior of out-of-time-order-correlators is intrinsically connected with fractional statistics in the topological order. We also propose a quantitative measure of chaos in a rational conformal field theory, which turns out to be determined by the topological entanglement entropy of the corresponding topological order.

Keywords: Anyons, Field Theories in Lower Dimensions, Topological Field Theories, Topological States of Matter

ArXiv EPrint: 1602.06543 


\section{Contents}

1 Introduction 1

2 Out-of-time-ordered-correlators in rational conformal field theories $\quad 3$

2.1 Definitions and conventions 3

2.2 An overview of existing results 4

2.3 Rational conformal field theories 5

2.4 Examples 8

3 The bulk-boundary correspondence 10

4 Out-of-time-ordered-correlators of random operators 13

$\begin{array}{lll}5 & \text { Conclusion and discussions } & 15\end{array}$

$\begin{array}{ll}\text { A Notations and conventions } & 16\end{array}$

B The monodromy matrix $\widetilde{M}[a, b] \quad 18$

C The residue value $r$ in $\mathrm{SU}(\mathrm{N})_{2}$ WZW models 20

\section{Introduction}

In classical chaos theory, the butterfly effect refers to the exponential sensitivity of the state of the system to initial conditions, which can be measured quantitatively by various quantities, such as the Lyapunov exponents. For example, denote one of the coordinates of the chaotic system as $x(t)$, which is a function of the initial conditions. The butterfly effect occurs for small perturbation to the initial position $x(0) \rightarrow x(0)+\delta x(0)$ if the change $|\delta x(t)| \propto e^{\lambda_{L} t}|\delta x(0)|$ grows exponentially in time, with $\lambda_{L}$ the Lyapunov exponent. The variation $\frac{\delta x(t)}{\delta x(0)}$ can be calculated by the Poisson braket $\{x(t), p(0)\}_{\mathrm{PB}}$. More generally, to obtain all Lyapunov exponents one should study the Poisson brakets $\left\{q_{i}(t), q_{j}(0)\right\}_{\mathrm{PB}}$ with $q_{i}$ different components of coordinates and canonical momenta.

The Poisson bracket formula suggests a natural generalization to quantum systems. In Heisenberg picture, quantum chaos can be characterized by the growth of operators of the form $i[x(t), p(0)]$. More precisely, for a given density matrix $\rho$ of a quantum particle, one should study $-\operatorname{Tr}\left(\rho[x(t), p(0)]^{2}\right)$, which measures the size of the commutator in the state $\rho .{ }^{1}$ (It should be noted that the square of the commutator should be considered since

\footnotetext{
${ }^{1}$ This type of measure of chaos first appears in the semi-classical treatment of a superconductor by Larkin and Ovchinnikov [1].
} 
we are interested in the size of the operator.) Recently, a generalization of such quantities have been studied in many-body systems, where operators $x$ and $p$ are replaced by generic many-body operators. [2-4]. If we take the thermal equilibrium state of the many-body system, the quantum butterfly effect refers to the increase of the thermal expectation value of the "commutator norm square":

$$
C(t):=\left\langle|[W(t), V(0)]|^{2}\right\rangle_{\beta}
$$

where $W(t)$ and $V(0)$ are generic Heisenberg operators at time $t$ and 0 . The thermal expectation value $\langle-\rangle_{\beta}$ is evaluated via the trace: $C(t)=Z^{-1} \operatorname{Tr}\left(e^{-\beta H}|[W(t), V(0)]|^{2}\right)$. When expanding the "commutator norm square," there are four terms in the function $C(t)$ :

$$
\begin{aligned}
& C(t)=\left\langle V^{\dagger}(0) W^{\dagger}(t)\right. W(t) V(0)+V^{\dagger}(0) W^{\dagger}(t) W(t) V(0) \\
&\underbrace{-W^{\dagger}(t) V^{\dagger}(0) W(t) V(0)-V^{\dagger}(0) W^{\dagger}(t) V(0) W(t)}_{\text {"Out-of-time-ordered correlators" }}\rangle_{\beta}
\end{aligned}
$$

The first two terms have the time order that appears in the response functions, therefore, are "accessible" [3]. However, the last two terms have a special "out-of-time-order" that is difficult to measure in conventional experiments. In a generic quantum many-body system, we expect the thermal averages of the accessible correlators to approach constant after the thermal time scale $\beta$; while the out-of-time-ordered correlators (OTOCs), possesses a nontrivial time dependence: it starts at large value and then decreases. The decrease of OTOCs corresponds to the increase of $C(t)$; therefore, it characterizes the quantum butterfly effect.

The OTOC has been extensively discussed in the connection between quantum gravity and quantum chaos, see ref. [2, 3, 5-7]. In those settings, for generic operators $W$ and $V$, the OTOC stays large until "scrambling time" [8-10] $t_{s c r}$, then it decreases rapidly to 0 and stays at 0 in late time $t \gg t_{s c r}$. Those behaviors are indicated by gravity, and are expected for a strongly chaotic quantum system. On the other hand, also interesting is the measurement of OTOC in "less chaotic" or "non-chaotic" models (e.g. see ref. $[11,12])$ to see how different aspects of the model affect the chaos. In this paper, we measure OTOCs in $(1+1)$-dimensional rational conformal field theories (RCFTs), ${ }^{2}$ which are known to be integrable. We focus on the late time regime $t \gg t_{s c r}$ and see how the "integrability" stops the late time value from vanishing in RCFTs. Remarkably, our result shows that when time $t$ goes to $\infty$, OTOCs in $(1+1)$-dimensional RCFTs are intrinsically related to the fraction statistics $[14,15]$ in $(2+1)$-dimensional topological order.

Fractional statistics and chaos are both interesting phenomena associated with nonlocal storage of information in strongly interacting quantum many-body systems. Fractional statistics usually arise in two-dimensional topological ordered states such as fractional quantum Hall states (FQH) [16, 17]. Theoretically, one can model the edge of FQH states using rational conformal field theories that possess the same algebraic structures/topological order as the corresponding FQH states. We use this type of bulk-

\footnotetext{
${ }^{2}$ For review, see ref. [13].
} 


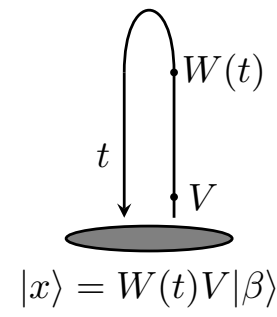

(a)

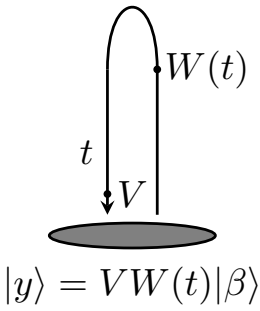

(b)

Figure 1. Illustration of the thermal expectation value of OTOC as an inner product of states $|x\rangle$ and $|y\rangle$. The operator $V$ and $W$ act on a pure state $|\beta\rangle$ (the grey disk) which is a purification of the thermal state. We can imagine $W(t)$ as a small perturbation in late time $t \gg \beta$ : if $W=$ Identity is trivial, then state $|x\rangle=|y\rangle$; if $W$ is a non-trivial perturbation and the system is "chaotic", we expect the "butterfly" $W$ causes a big difference on states $|x\rangle$ and $|y\rangle$. Therefore, we can use the inner product $\langle y \mid x\rangle$ to quantify the butterfly effect.

boundary correspondence ${ }^{3}$ to build the connections between the butterfly effect on the boundary and the fractional statistics in the bulk.

In section 2, we present the CFT computation of OTOC in the content of RCFTs, following the technique developed in ref. [18], and show that the OTOC at $t=\infty$ only depends on the modular S-matrix. Section 2 also includes more examples and discussion of small late time values. Section 3 is devoted to an alternative derivation of the result via bulk-boundary correspondence, which shows the non-trivial "topology" behind the OTOC. We also consider the OTOC between "random operators" in section 4, where we show an unexpected connection between the boundary butterfly effect and the bulk topological entanglement entropy. Section 5 contains conclusions and discussions on real condensed matter experiments. Most of the technical details are placed in the appendices.

\section{Out-of-time-ordered-correlators in rational conformal field theories}

\subsection{Definitions and conventions}

Before going to the detailed discussions, we first declare some general definitions for OTOC. More specifically, we are considering thermal expectation value of OTOC in a quantum system with Hilbert space $\mathcal{H}: f(t):=\left\langle W^{\dagger}(t) V^{\dagger}(0) W(t) V(0)\right\rangle_{\beta}, V$ and $W$ act on $\mathcal{H}$. Alternatively, one can interpret $f(t)$ as an inner product between two pure states (see figure 1 ):

$$
f(t)=\langle y \mid x\rangle ; \quad|x\rangle=W(t) V|\beta\rangle,|y\rangle=V W(t)|\beta\rangle
$$

where $|\beta\rangle$ is a purification of the thermal system at temperature $T=1 / \beta$, e.g., a thermofield double state $[19,20]|\beta\rangle=Z^{-1 / 2} \sum_{n} e^{-\beta E_{n} / 2}|n\rangle|\bar{n}\rangle \in \mathcal{H} \otimes \overline{\mathcal{H}}$. We require all operators act on one side, say $\mathcal{H}$.

\footnotetext{
${ }^{3}$ This is the type of correspondence between $(1+1)$-dimensional RCFTs and $(2+1)$-dimensional TQFTs, e.g. WZW/CS correspondence. One should avoid confusing with the AdS/CFT correspondence.
} 
As a measure of the difference between $|x\rangle$ and $|y\rangle$, it is convenient to use the normalized value:

$$
\widetilde{f}(t):=\frac{\langle y \mid x\rangle}{\sqrt{\langle x \mid x\rangle\langle y \mid y\rangle}}
$$

Previous studies $[2,3,7,18]$ of such correlation function focused on the early time $\beta<$ $t<t_{s c r}$, i.e., between the dissipation time and the scrambling time, and found interesting "Lyapunov behavior" ${ }^{4}$ for those systems that can be holographically described by Einstein gravity. However, for a generic system that does not have a large separation between dissipation time $t_{d} \sim \beta$ and scrambling time $t_{s c r}$, the "Lyapunov behavior" is not welldefined. Instead, one can focus on the later time regime $t \gg t_{s c r}$, which characterized the residue part of the system that "survived" under the butterfly effect.

For later convenience, we denote the inner product $\langle x \mid x\rangle=\left\langle V^{\dagger} W^{\dagger}(t) W(t) V\right\rangle_{\beta}$ by $g(t)$. In the regime $t \gg \beta$, such a four point function generically factorizes to $g(t) \sim$ $\left\langle V^{\dagger} V\right\rangle_{\beta}\left\langle W^{\dagger} W\right\rangle_{\beta}$, which represents a normalization for operators $W$ and $V$. The same applies to $\langle y \mid y\rangle$. In the following section, we will study the late time behavior of $\widetilde{f}(t)$ in the context of RCFTs.

\subsection{An overview of existing results}

As a starting point of our discussion, we briefly review the calculation of OTOC in (1+1)-d CFT in ref. [18].

To setup the CFT computation, it is essential to use complexified time $t_{c}=t-i \tau$, where $t$ stands for the Minkowski time, and $\tau$ for the Euclidean time. The strategy is to begin with a pure Euclidean computation with $t=0$ and then analytically continue it to the desired Minkowski time $t$. Such strategy enables us to manipulate the order of operators by tuning the auxiliary small imaginary part $\tau=\epsilon$. After the conformal mapping $z=\exp (2 \pi w / \beta), \quad \bar{z}=\exp (2 \pi \bar{w} / \beta)$, with $w=x-t_{c}$ and $\bar{w}=x+t_{c}{ }^{5}$ the thermal expectation value is mapped to the vacuum expectation value. In the end, it is essential to consider the vacuum expectation value of the four point function which has a general decomposition in terms of conformal blocks [18, 21, 22]

$$
\left\langle W^{\dagger}\left(z_{1}, \bar{z}_{1}\right) W\left(z_{2}, \bar{z}_{2}\right) V^{\dagger}\left(z_{3}, \bar{z}_{3}\right) V\left(z_{4}, \bar{z}_{4}\right)\right\rangle=\frac{1}{z_{12}^{2 h_{w}} z_{34}^{2 h_{v}}} \frac{1}{\bar{z}_{12}^{2 \bar{h}_{w}} \bar{z}_{34}^{2 \bar{h}_{v}}} \sum_{p, \bar{p}} g_{p, \bar{p}} \mathcal{F}_{p}(\eta) \overline{\mathcal{F}}_{\bar{p}}(\bar{\eta})
$$

where $z_{i j}=z_{i}-z_{j}$, and $\eta=\frac{z_{12} z_{34}}{z_{13} z_{24}}, \bar{\eta}=\frac{\bar{z}_{12} \bar{z}_{34}}{\bar{z}_{13} \bar{z}_{24}}$ are cross ratios, $g_{p, \bar{p}}$ is the pairing coefficient for holomorphic block $p$ and anti-holomorphic block $\bar{p}$. It is important to note that the summation is only over conformal families labeled by $\{p, \bar{p}\}$ rather than all the primaries and descendants individually. For the parameter regime $t \gg \beta$ that we are interested in,

\footnotetext{
${ }^{4}$ Here Lyapunov behavior refers to the functional dependence of $f(t)$ on $t$ at early time:

$$
f(t) \sim 1-\frac{1}{N} e^{\lambda_{L} t}+\ldots
$$

where $N$ is some big number in the model, and the exponent $\lambda_{L}$ is recognized as Lyapunov exponent. Physically, the Lyapunov behavior characterizes how fast the chaos develops in the system.

${ }^{5}$ We use a different sign convention comparing to ref. [18] for later convenience.
} 
the cross ratio is always small: $\eta, \bar{\eta} \sim \exp (-2 \pi t / \beta) \ll 1$. Therefore, we can formally expand $\mathcal{F}_{p}(\eta)$ according to the powers of $\eta$ :

$$
\mathcal{F}_{p}(\eta)=\sum_{n=0}^{\infty} F_{p, n} \eta^{h_{p}+n}, \quad \text { if } N_{a \bar{a}}^{p} N_{b \bar{b}}^{p} \neq 0
$$

where $h_{p}$ denotes the dimension of the primary $p$ (and $h_{p}+n$ for descendants, $n \geq 1$ ). $\left\{F_{p, n}\right\}$ are coefficients depending on the details of operator $W$ and $V . a, b$ label the conformal families of $W, V$, correspondingly. (For simplicity we can assume $W, V$ are primaries. We sometimes make the family label explicit by denoting $W=W[a]$ and $V=V[b]$.) The fusion multiplicity $N_{a \bar{a}}^{p}$ counts the dimension of operator product algebra from $a, \bar{a}$ to intermediate channel $p . \mathcal{F}_{p}=0$ if $N_{a \bar{a}}^{p} N_{b \bar{b}}^{p}=0$, i.e., if $p$ is absent in the fusion channels of either $a \times \bar{a}$ or $b \times \bar{b}$.

The behavior of OTOC is determined by the dependence of conformal blocks on cross ratio $\eta$ : for arbitrary four points on complex plane, it is convenient to use a conformal map to move three of them to standard positions: 0,1 and $+\infty$, and leave over with a free parameter $\eta$. Therefore, the conformal blocks $\left\{\mathcal{F}_{p}(\eta)\right\}$ will have three singularities in general: $\eta=0,1$ and $\infty$ (see figure 2a). The same is true for $\overline{\mathcal{F}}(\bar{\eta})$. When implementing the analytic continuation to Minkowski time $t$, the path $\eta(t)$ might go around a singular point and leave from the principal sheet to the second sheet. One can check (e.g. see ref. [18]) that among conformal blocks in the OTOC $f(t)$, only the holomorphic $\eta$ winds a topologically nontrivial loop, ${ }^{6}$ as is shown in figure 2a. In contrast, the winding of $\eta$ or $\bar{\eta}$ is trivial in the in-timeordered correlator $g(t)$. This is the key difference that leads to a nontrivial ratio $\widetilde{f}(t)$.

\subsection{Rational conformal field theories}

For general CFTs, the number of conformal families can be infinite and there is not much general information we can tell. One can instead restrict to certain subclass of CFTs. For example in ref. [18], the authors analyzed the behavior of the Virasoro identity block of a holographic CFT [23-25] and deduced a result about the butterfly effect that is consistent with the holographic analysis. ${ }^{7}$ In this paper, we will choose to work in another rich subclass of CFTs - diagonal rational conformal field theories, which have a well-controlled algebraic structure on conformal blocks (for a review of RCFTs, see ref. [13] and reference therein). In this subclass, the four point functions in Euclidean time have a simpler finite sum presentation:

$$
\left\langle W^{\dagger}\left(z_{1}, \bar{z}_{1}\right) W\left(z_{2}, \bar{z}_{2}\right) V^{\dagger}\left(z_{3}, \bar{z}_{3}\right) V\left(z_{4}, \bar{z}_{4}\right)\right\rangle=\frac{1}{z_{12}^{2 h_{w}} z_{34}^{2 h_{v}}} \frac{1}{\bar{z}_{12}^{2 \bar{h}_{w}} \bar{z}_{34}^{2 \bar{h}_{v}}} \sum_{i=1}^{N} \mathcal{F}_{i}(\eta) \overline{\mathcal{F}}_{i}(\bar{\eta})
$$

\footnotetext{
${ }^{6}$ Technically speaking, to make the statement more precise, one need to place $W^{\dagger}$ and $W$ at $x \gg \beta$, s.t. $\eta$ starts at vicinity of 0 . This specific setting is not essential to the results in this paper. The key point is that the difference between path $\eta(t)$ and $\bar{\eta}(t)$ is a nontrivial winding around singularity at $z=1$, which is always valid for $f(t)$ at late time regardless of the initial position of operators.

${ }^{7}$ To avoid confusion, we clarify that the previous studies $[2,3,18]$ were mainly interested in the Lyapunov exponent and scrambling time, which are in the "early time" regime, while the current work will focus on the "late time" regime instead.
} 


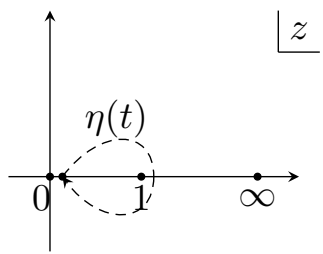

(a) Monodromy (analytic).
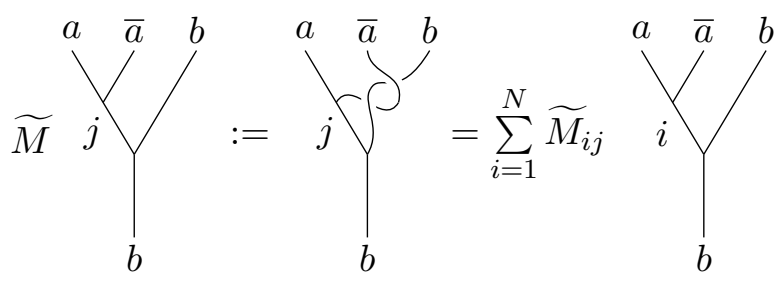

(b) Monodromy (algebraic).

Figure 2. (a) Winding $\eta(t)$ in the moduli space: $\eta(t) \in \mathbb{C}-\{0,1,+\infty\}$ winds around $z=1$ clockwisely and induces a linear transformation on the space of conformal blocks $V_{\bar{a} a \bar{b} b} \simeq V_{b}^{a \bar{a} b}$, which defines the the monodromy matrix. (b) The diagrammatic representation of the monodromy matrix $\widetilde{M}=\widetilde{M}[a, b]$. For fixed $a, b, \widetilde{M}[a, b]$ acts by braiding the two lines $\bar{a}, b$. See appendix A for detailed conventions of the diagrammatics.

where holomorphic conformal blocks $\left\{\mathcal{F}_{i}\right\}$ form a finite dimensional vector space: $V_{\bar{a} a \bar{b} b} \cdot{ }^{8}$ This linear space is parametrized by the cross ratio $\eta$, and the same applies to the antiholomorphic $\overline{\mathcal{F}}_{i}$ and $\bar{\eta} \cdot{ }^{9}$

In this setting, we are able to discuss the effect of analytic continuation to the second sheet more concretely: according to the general principle of RCFTs [27], a full winding around a singularity induces a linear transformation $\widetilde{M}[a, b]$ in space $V_{\bar{a} a \bar{b} b}$, known as the monodromy [21]. See figure 2 for its definition in terms of diagrams.

With this definition, one can compute the matrix element of $\widetilde{M}$ in terms of the general algebraic data of RCFT, known as the F-matrix and the R-matrix. This computation is well-known in the literature, and we include a summary of it in the appendix B. The monodromy matrix is determined by universal data that only depends on the algebraic structure of RCFTs, independent of the particular state chosen in the family and the details of locations of the operators. Now with $\widetilde{M}_{i j}$ defined in figure 2 , we can rewrite the formula of normalized OTOC more explicitly:

$$
\widetilde{f}(t)=\frac{\sum_{i, j=1}^{N} \widetilde{M}_{i j} \mathcal{F}_{i}(\eta) \overline{\mathcal{F}}_{j}(\bar{\eta})}{\sum_{i=1}^{N} \mathcal{F}_{i}(\eta) \overline{\mathcal{F}}_{i}(\bar{\eta})}
$$

the normalization cancels the unimportant common prefactor $\frac{1}{z_{12}^{2 h} z_{34}^{2 h_{v}}} \frac{1}{z_{12}^{2 h_{w}} \bar{z}_{34}^{2 h_{v}}}$. Nevertheless, the actual functional form of $\widetilde{f}(t)$ is still messy because of the appearance of descendants when one explicitly expand the conformal blocks as power series of $\eta$. However, the result is simplified in the late time $t \gg \beta$ regime, with $\eta, \bar{\eta} \sim \exp (-2 \pi t / \beta)$.

\footnotetext{
${ }^{8}$ Here we use an index $i$ instead of the intermediate channel label $p$ due to the subtlety that a fixed channel $p$ might have dimension $N_{a \bar{a}}^{p} N_{b \bar{b}}^{p}>1$. Later we will ignore this subtlety and use indices $i, j$ to label the intermediate channels as well. Alternatively, one can treat channel labels as labeling a subspace instead of a vector.

${ }^{9}$ Alternatively, one can formulate conformal blocks as vector bundle over the moduli space [26]. The monodromy is defined as that of the vector bundle. Such a vector bundle is also equipped with a fiber-wise metric for computing physical correlation functions. In the diagonal theory here, the metric is $\delta_{i j}$.
} 
First of all, the residue value at $t \rightarrow \infty$ is free of complicated coefficients (i.e., those $\left.F_{i, n} \mathrm{~S}\right)$ :

$$
r[a, b]=\lim _{t \rightarrow \infty} \widetilde{f}(t)=\frac{\widetilde{M}[a, b]_{11}\left|\mathcal{F}_{1}(0)\right|^{2}}{\left|\mathcal{F}_{1}(0)\right|^{2}}=\widetilde{M}[a, b]_{11}
$$

only depends on universal data: $(1,1)$ component of monodromy matrix $\widetilde{M}[a, b]$. Interestingly, the $(1,1)$ element of monodromy matrix can be expressed solely by the modular S-matrix (see appendix B for a more detailed derivation):

$$
\widetilde{M}[a, b]_{11}=\frac{\mathcal{D} s_{a b}^{*}}{d_{a} d_{b}}=\frac{s_{11} s_{a b}^{*}}{s_{1 a} s_{1 b}}
$$

where $\mathcal{D}=1 / s_{11}$ is the total quantum dimension, and $d_{a}=s_{1 a} / s_{11}$ are the quantum dimensions of individual conformal families. (As a side remark, $\widetilde{M}[a, b]_{11}$ is known as monodromy scalar in the literature of anyon interferometry [28, 29], which we will discuss more in later part of the article.)

From the equation (2.8), one can immediately see that Abelian theories ${ }^{10}$ have $|r|=1$. In general, one can prove a physically intuitive inequality $|r| \leq 1$ by noticing

$$
\left|s_{a b}\right|=\frac{1}{\mathcal{D}}\left|\sum_{c} d_{c} N_{a b}^{c} \frac{\theta_{c}}{\theta_{a} \theta_{b}}\right| \leq \frac{1}{\mathcal{D}} \sum_{c} d_{c} N_{a b}^{c}=\frac{d_{a} d_{b}}{\mathcal{D}}
$$

Therefore, $|r|=\mathcal{D}\left|s_{a b}\right| / d_{a} d_{b} \leq 1$. When there is only one fusion channel in $a \times b$, i.e. when $N_{a b}^{c}$ is nonzero for only one $c$, the inequality picks equal sign.

From this somewhat "trivial" inequality, we see an interesting indication: "scrambling", ${ }^{11}$ which refers to the suppression of $r$ here, only comes from non-Abelian anyons. Physically, the scrambling occurs due to nontrivial interference between different fusion channels, which describes the spreading of quantum information more and more non-locally among different conformal families. For Abelian anyons, the long-time value of OTOC is only different from the short-time value by a phase. Although such a phase is still a topological feature, it does not lead to scrambling since there is no nontrivial unitary transformation in the vector space generated. This observation is closely related to the correspondence between scrambling and fractional statistics, which we will elaborate in next section.

Now we return to more general components of matrix $\widetilde{M}$, which appears in the form of $\widetilde{M}_{i j} \eta^{h_{i}+n} \bar{\eta}^{h_{j}+m}$ in the numerator of equation (2.6). $\quad n, m \in \mathbb{Z}^{\geq 0}$ come from possible descendants. As a function of $t$, such terms decay exponentially as $\widetilde{M}_{i j} \exp \left(-2 \pi\left(h_{i}+h_{j}+m+n\right) t / \beta\right)$. Except the $(1,1)$ element we mentioned before, it is generally unpractical to separate a prefactor from an exponential decaying function. However, we can still make non-trivial prediction on the "spectroscopy" of function $\tilde{f}(t)$ by knowing whether certain $\widetilde{M}_{i j}$ is non-zero. The exponents from the denominator:

\footnotetext{
${ }^{10}$ We borrow the terminology from anyon theory $[30,31]$ that non-Abelian anyons $a, b$ refer to those conformal families having multiple fusion channels e.g. $a \times b=c+d+\ldots$, and Abelian theories refer to a theory without non-Abelian anyons.

${ }^{11}$ Scrambling is defined as the process where a non-entangled state evolves into an almost maximally entangled state, or correspondingly, the process of a simple local operator evolves into a complicated operator that is supported on almost the whole system. For details see ref. [2, 8-10].
} 


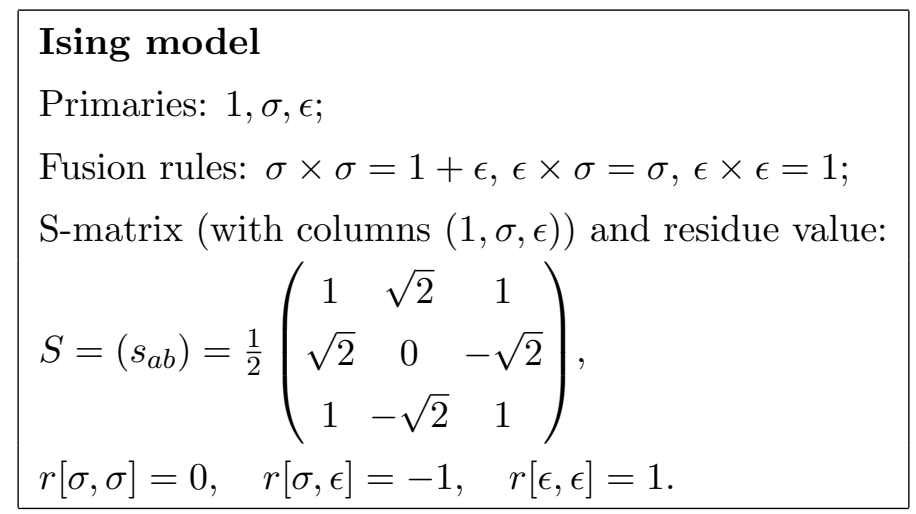

Table 1. Algebraic data of Ising model. Residue value $r$ is computed according to equation (2.7) and (2.8).

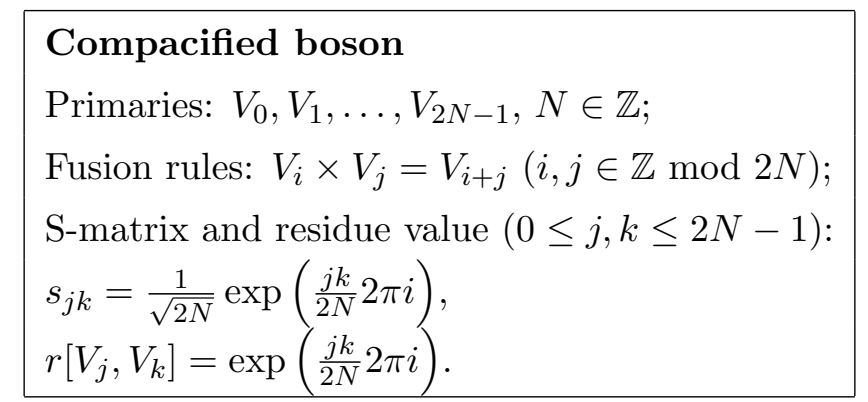

Table 2. Algebraic data of compactified boson. Residue value $r$ is computed according to equation (2.7) and (2.8).

$\sum_{i=1}^{N} \mathcal{F}_{i}(\eta) \overline{\mathcal{F}}_{i}(\bar{\eta})$ is always in form of $\Delta_{i, n}=2 h_{i}+n, n \in \mathbb{Z}^{\geq 0}$ as a consequence of diagonal pairing. However, the numerator: $\sum_{i, j=1}^{N} \widetilde{M}_{i j} \mathcal{F}_{i}(\eta) \overline{\mathcal{F}}_{j}(\bar{\eta})$ contains mixed exponents $\Delta_{i, j, n}=h_{i}+h_{j}+n$, which enriches the spectrum. In particular, the slowest decaying rate comes from pairing between identity block with the smallest scaling dimension $h_{z}$ (assuming $h_{z}<1$ ), if the corresponding matrix element $\widetilde{M}_{1 z}$ (or $\widetilde{M}_{z 1}$ ) is non-vanishing. Such elements of the monodromy matrix are related to the "generalized" modular S-matrix $s_{\bar{z}, a \bar{b}}$ for one-punctured-torus [30, 32] as $\widetilde{M}_{1 z}=\frac{\mathcal{D} s_{z, a \bar{b}}}{d_{a} d_{b}}$. More details are presented in appendix B.

\subsection{Examples}

To make the abstract discussion more transparent, in this section we present three examples: (1) Ising model, see table 1; (2) Compactified boson, see table 2; and (3) SU(2) WZW model at level $k$, see table 3 .

The Ising model was considered as an example of non-chaotic theory in ref. [18]. Our result on late time residue value is consistent with ref. [18]. Method here has the advantage that it only relies on a small amount of universal data rather than the explicit functional form of conformal blocks or four point functions.

The compactified boson is an example of Abelian theory. The residue value for this model is a pure phase $|r|=1$ since there is no non-Abelian anyon. 


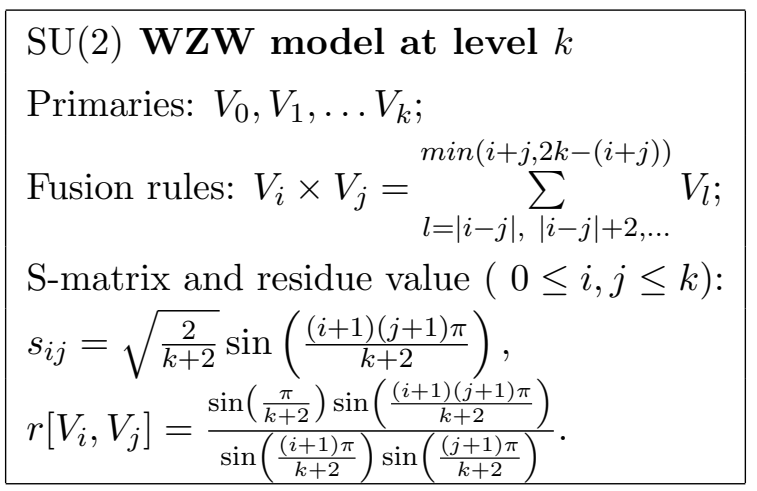

Table 3. Algebraic data of SU(2) WZW model $[33,34]$ at level $\mathrm{k}$. Residue value $r$ is computed according to equation (2.7) and (2.8).
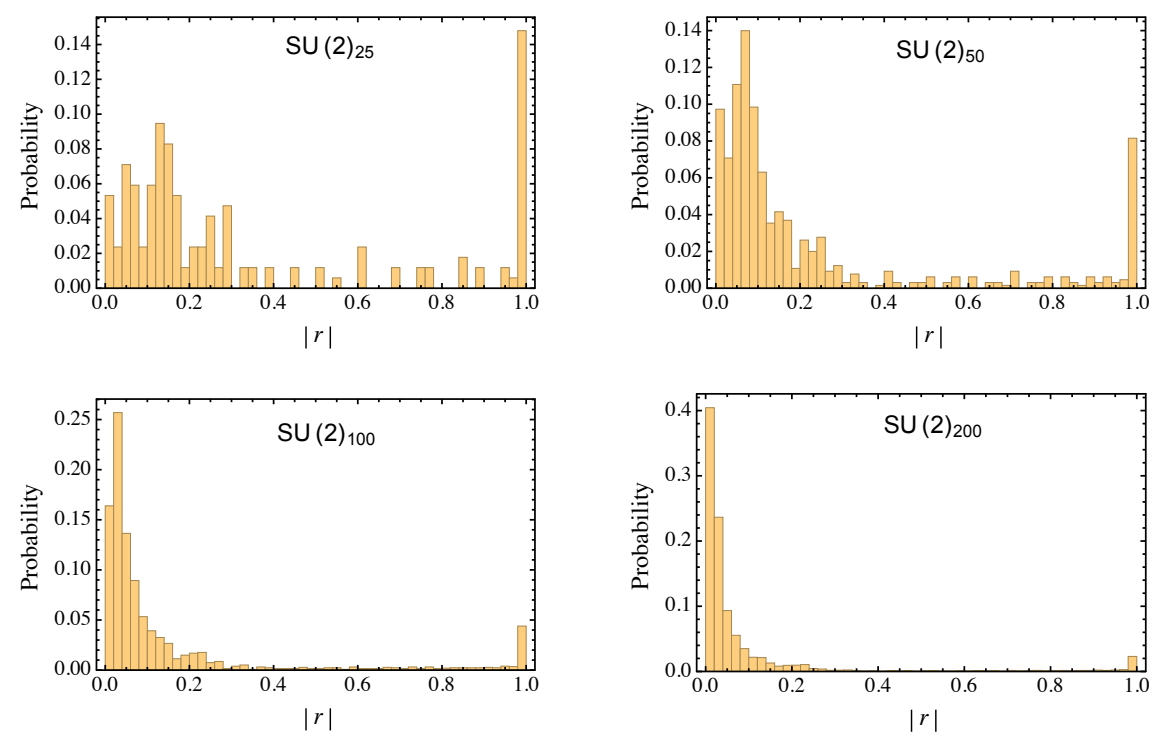

Figure 3. The distributions of $\left|r\left[V_{i}, V_{j}\right]\right|$ in $\mathrm{SU}(2)$ WZW models.

In the example of WZW models, residue value $r$ is strongly oscillating in $i$ and $j$, so that some channels are more scrambled than others. To understand the overall behavior of the magnitude of $r$, which describes the strength of "scrambling", we show a probability distribution plot of $\left|r\left[V_{i}, V_{j}\right]\right|$ for the $\mathrm{SU}(2)_{k}$ WZW model at level $k=25,50,100,200$ in figure 3. One can see from the distribution that the theory is more chaotic in larger $k$, where most of the $|r|$ is close to zero.

In appendix C, we present the statistics of $|r|$ for a related family of RCFT's, the SU( $k$ ) WZW models at level 2. These theories are related to $\mathrm{SU}(2)_{k}$ by the level-rank duality [22], but they have a large central charge $c=\frac{2\left(k^{2}-1\right)}{k+2}$. We prove there in appendix $\mathrm{C}$ that the statistics of $|r|$ in $\mathrm{SU}(k)_{2}$ is identical to that of $\mathrm{SU}(2)_{k}$, which makes them interesting examples of RCFT's with stronger chaos in the large central charge limit. 


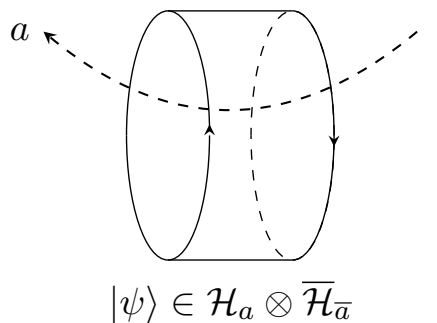

(a) Fixed time slide.

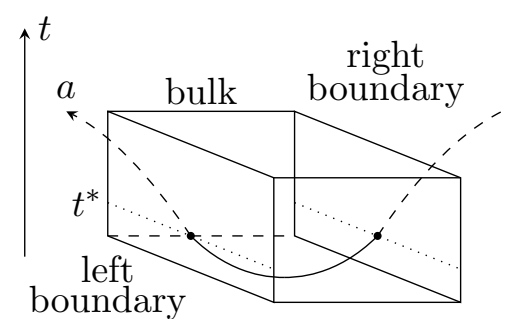

(b) Space-time picture.

Figure 4. The bulk-boundary correspondence. (a) At a fixed time $t^{*}$, the CFT state at two boundaries belong to sector $\mathcal{H}_{a} \otimes \mathcal{H}_{\bar{a}}$; (b) The spacetime picture for a pair of anyons $a, \bar{a}$ created and passed through the boundary at fixed time $t^{*}$. The state was in identity sector before $t^{*}$ : $\psi\left(t<t^{*}\right) \in \mathcal{H}_{1} \otimes \overline{\mathcal{H}}_{1}$, and shifted to sector $\mathcal{H}_{a} \otimes \overline{\mathcal{H}}_{\bar{a}}$ after time $t^{*}$.

\section{The bulk-boundary correspondence}

As can be seen from the discussion in the previous section, the language of anyons (more precisely, the language of unitary modular tensor category (UMC)) is useful in discussing RCFTs, since they share the same algebraic structure. In this section, we will propose a physical setup to demonstrate that the correspondence between scrambling in RCFTs and anyons in $(2+1)$-dimensions is not a formal connection, but has a intrinsic physical reason, coming from the bulk-boundary correspondence of $(2+1)$-d TOS.

Chiral part of RCFT (i.e. the holomorphic sectors, or the anti-holomorphic sectors) can be realized as the edge theory of $(2+1)$-d chiral topological order $[13,35]$, such as fractional quantum Hall $(\mathrm{FQH})$ states $[36,37]$. In this bulk-boundary correspondence, a non-chiral RCFT can be viewed as the low energy effective theory of a strip of $(2+1)$-d chiral topological order, as is shown in figure 4. In such a strip, the edge states on the two boundaries are described by the holomorphic and anti-holomorphic sectors of a RCFT, and the bulk topological order is described by the corresponding UMC. There is a one-to-one correspondence between the labels of primary fields in RCFT and the labels of anyon types in the bulk. When the bulk has no anyon (or has anyons that fuse to identity, i.e., have zero total anyonic charge), the topological sector of the two boundaries must be conjugate of each other, so that the low energy Hilbert space of this system is the same as that of a diagonal RCFT:

$$
\mathcal{H}_{\text {total }}=\bigoplus_{a} \mathcal{H}_{a} \otimes \overline{\mathcal{H}}_{\bar{a}}
$$

One can also consider the physical process in the $(2+1)$-d strip corresponding to acting operators in the RCFT in the spacetime picture, see figure $4 \mathrm{~b}$. The insertion of a primary operator of family $(a, \bar{a})$ at $\left(x, t^{*}\right)$ on the boundary corresponds to creating a pair of anyons $(a, \bar{a})$ at an earlier time and pass them through the boundary at spacetime point $\left(x, t^{*}\right)$. To compute two point functions, one need to get them back at a later time and annihilate them in the bulk.

For four point functions, the procedure is similar. However, we will show in details that there is a nontrivial linking structure in the bulk picture for OTOC. In our two-side 


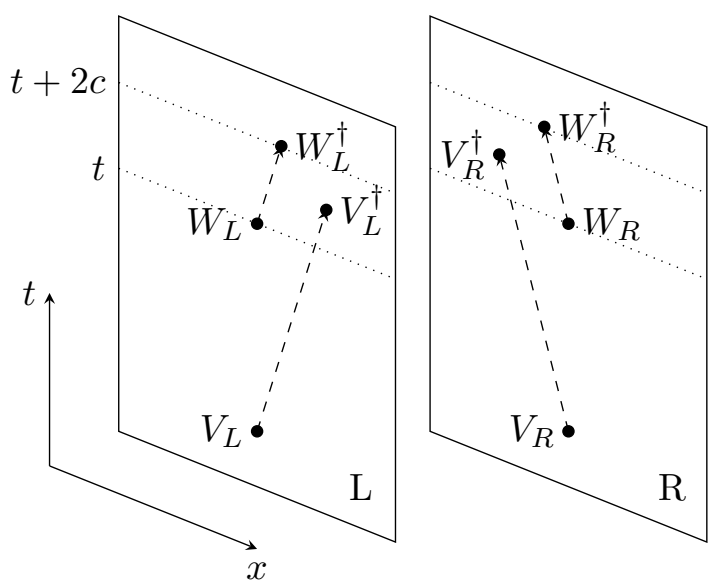

Figure 5. OTOC is mapped to a time-ordered four-point function on the two boundaries of a strip, by shifting along the light cones (see equation (3.2).

setting, one can split each physical operator into left-moving and right-moving parts, acting on the left and right boundaries of the strip: $\mathcal{O}(x, t)=\mathcal{O}_{L}(x, t) \mathcal{O}_{R}(x, t)$. The advantage of such a decomposition is that we are allowed to move the chiral operators along light cones freely: $\mathcal{O}_{L}(x, t)=\mathcal{O}_{L}(x-c, t-c)$ and $\mathcal{O}_{R}(x, t)=\mathcal{O}_{R}(x+c, t-c)$ where $c$ is an arbitrary constant. Therefore, we can use this freedom to map the OTOC to a time-ordered four-point function:

$$
\begin{aligned}
f(t)=\left\langle W(t)^{\dagger} V^{\dagger} W(t) V\right\rangle_{\beta}= & \left\langle W_{L}^{\dagger}(2 c, t+2 c) V_{L}^{\dagger}(t+c, t+c) W_{L}(0, t) V_{L}(0,0) .\right. \\
& \left.\times W_{R}^{\dagger}(-2 c, t+2 c) V_{R}^{\dagger}(-t-c, t+c) W_{R}(0, t) V_{R}(0,0)\right\rangle_{\beta}
\end{aligned}
$$

with $c>0$. After this shift, we can compute the OTOC in time ordered way, as shown in figure 5 .

From the viewpoint of bulk-boundary correspondence (figure 6a), the insertions of operators $V[b], V^{\dagger}[\bar{b}]$ on two boundaries corresponds to creating a pair of anyons labeled by $b, \bar{b}$ and passing through the boundary at insertion points of $V_{L}, V_{R}$, then pull the anyons back at the insertion points of $V_{L}^{\dagger}, V_{R}^{\dagger}$. Similar discussion applies to operators $W[a], W^{\dagger}[\bar{a}]$. Remarkably, this configuration of operators on the boundaries induce a nontrivial linking of anyon worldlines in the bulk, as is shown in figure 6a. One can further compute the linked world lines in the anyon theory, which gives $\mathcal{D} s_{a b}^{*}$. The computation is under the assumption that bulk energy gap of topological quantum matter is much larger than the temperature $1 / \beta$ such that we can ignore the actual dynamics of the bulk particles and only focus on the topological content.

Alternatively, one can interpret the bulk-boundary correspondence here by starting with a pair of world-lines for anyons $a$ and $b$, see figure $6 \mathrm{~b}$, and then imagine to move the boundaries inwards. The boundaries will finally intersect with the world lines, which induce insertions of operators on the boundaries. If we start with the linked configuration as shown in figure $6 \mathrm{~b}$, we are able to arrange the crossing points such that they correspond to the OTOC in equation (3.2). 


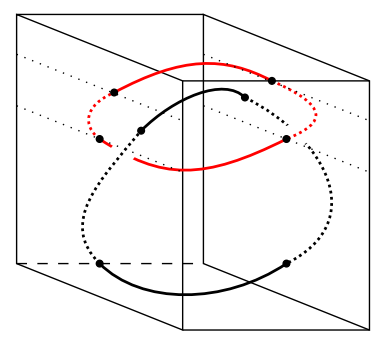

(a)

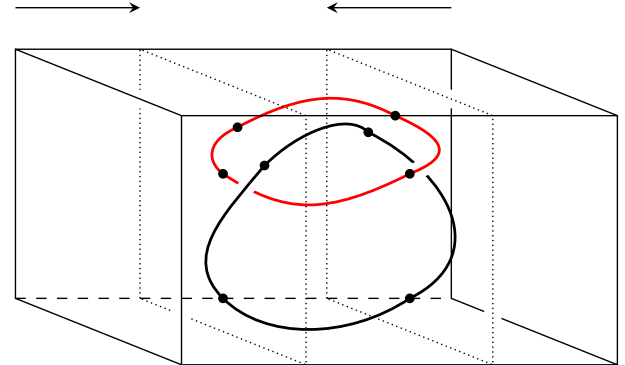

(b)

Figure 6. (a) Anyon world-lines corresponding to the OTOC. Black lines (solid for interior, dotted for exterior) are for anyon label $b$, and red lines are for anyon label $a$. The insertion point at the boundary is determined by equation (3.2) and figure 5. (b) The interpolation between a completely bulk anyon braiding procedure and a boundary OTOC. Starting with a pair of linked anyon worldlines inside the bulk, one can move both boundaries inwards to intersect with the world-lines. Such world lines will induce insertion of operators in the families $a, b$. By proper arrangement of initial configurations of the linked world lines, one obtains the OTOC in subfigure (a).

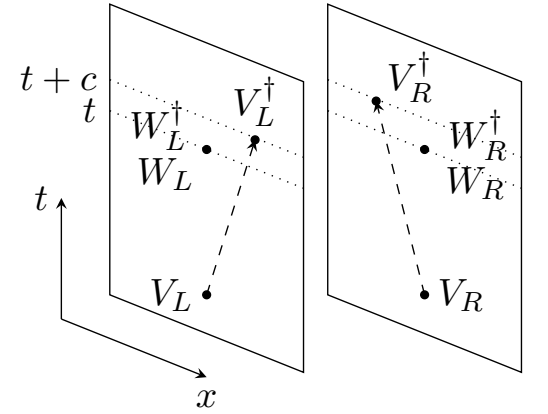

(a) Boundary.

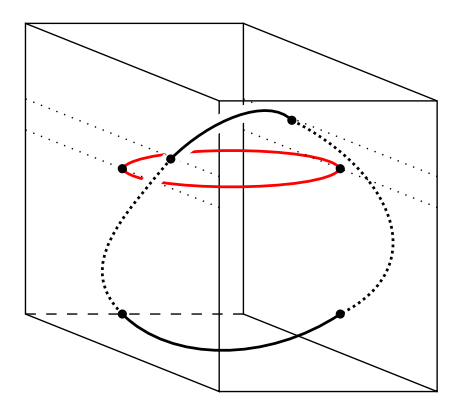

(b) Bulk.

Figure 7. Bulk-boundary correspondence for ordinary ordered four point function $g(t)$. (a) shows the insertion of operators $V[b], W[a]$.. on two boundaries of a strip of topological quantum matter, we shifts the operator along light cone to reach the position in equation (3.3). (b) shows the corresponding anyon world-lines, red for label $a$ and black for label $b$, such world-line configuration doesn't link, therefore can be deform to two independent loops. (Notice that $W_{L / R}$ and $W_{L / R}^{\dagger}$ share the same location in figures.)

As a comparison, one can run the same procedure for ordinary ordered correlation function $g(t)$, which can be split and shifted to time ordered as well:

$$
\begin{aligned}
g(t)=\left\langle V^{\dagger} W^{\dagger}(t) W(t) V\right\rangle_{\beta}= & \left\langle V_{L}^{\dagger}(t+c, t+c) W_{L}^{\dagger}(0, t) W_{L}(0, t) V_{L}(0,0) .\right. \\
& \left.\times V_{R}^{\dagger}(-t-c, t+c) W_{R}^{\dagger}(0, t) W_{R}(0, t) V_{R}(0,0)\right\rangle_{\beta}
\end{aligned}
$$

The bulk picture for $g(t)$ was shown in figure $7 \mathrm{~b}$, where we can clearly see that the world lines are unlinked, which therefore, correspond to two independent loops with labels $a$ and $b$. Such loops together have the amplitude $d_{a} d_{b}$ according to theory of anyons.

In summary, we have provided a physical interpretation to the correspondence between late-time universal behavior of OTOC in RCFT and fractional statistics of anyons 
in $(2+1)$-d TOS. The intrinsic reason of this correspondence is the fact that these two seemingly unrelated phenomena - the butterfly effect and fractional statistics - are both consequences of nonlocality in unitary time evolutions. The butterfly effect comes from the propagation of quantum information in the Hilbert space from simple local operators to more and more non-local operators (for related discussion see ref. [38]) which makes it more and more difficult to reveal the information locally. Similarly, fractional statistics is only possible because anyons are intrinsically non-local. The braiding of anyons, especially that of non-Abelian anyons, lead to a nontrivial and non-local unitary transformation on the Hilbert space, which can be viewed as a special example of scrambling.

\section{Out-of-time-ordered-correlators of random operators}

In the previous sections, we have discussed how OTOC of a pair of operators, each in a prefixed conformal family, depends on the algebraic content of the operators. Indeed, by the bulk-boundary correspondence, we show that OTOC in RCFT is related to the braiding of anyons in $(2+1)$-d. In this section, we would like to investigate how OTOC can be used as a diagnostic of RCFTs. For that purpose, we would like to consider OTOC of a generic pair of operators, rather than selecting particular operators by hand. A natural choice is to consider OTOC between two randomly chosen operators. The key question we need to address is what is a natural random ensemble of operators in an RCFT.

To specify the random ensemble, we need a proper probability distribution for conformal families. Since the Hilbert space of RCFT is a direct sum of that of each conformal family (equation (3.1)), the probability of a random vector in the Hilbert space to be in a given conformal family is proportional to the Hilbert space dimension of that family, i.e., $p_{a} \propto \operatorname{dim}\left(\mathcal{H}_{a} \otimes \overline{\mathcal{H}}_{\bar{a}}\right)$. Naively this is not meaningful, since the Hilbert space dimension of each family is infinite. Nevertheless, the ratio of the Hilbert space dimensions of different sectors is well-defined and is determined by the quantum dimension:

$$
\frac{\operatorname{dim} \mathcal{H}_{a}}{\operatorname{dim} \mathcal{H}_{1}}=\frac{\chi_{a}(0)}{\chi_{1}(0)}=\frac{s_{a 1}}{s_{11}}=d_{a}
$$

where $\chi_{a}(\tau)=\operatorname{Tr}_{a} e^{2 \pi i \tau\left(L_{0}-c / 24\right)}$ is the character for sector $a . \chi_{a}(0)$ is the high temperature limit of the character. ${ }^{12}$ Since only the ratio between different sectors matter for the definition of random ensemble, we can define a regularized probability $p_{a}=d_{a}^{2} / \mathcal{D}^{2}$. When we consider a random operator, it is chosen to be in sector $(a, \bar{a})$ with probability $p_{a}$.

It is interesting to note that this probability distribution is also fusion invariant, i.e., $\sum_{a, b} p_{a} p_{b} p_{a b \rightarrow c}=p_{c}$, where $p_{a b \rightarrow c}$ is the probability of fusing $a$ and $b$ to total charge $c$. According to ref. [40], $p_{a b \rightarrow c}=d_{c} N_{a b}^{c} / d_{a} d_{b}$. Therefore,

$$
\sum_{a, b} p_{a} p_{b} p_{a b \rightarrow c}=\sum_{a, b} \frac{d_{a}^{2} d_{b}^{2}}{\mathcal{D}^{4}} \frac{d_{c} N_{a b}^{c}}{d_{a} d_{b}}=\frac{\sum_{b} d_{b}^{2} d_{c}^{2}}{\mathcal{D}^{4}}=p_{c}
$$

\footnotetext{
${ }^{12} \chi_{a}(0)$ can be computed by the modular $S$ matrix: $\chi_{a}(\tau)=\sum_{b} s_{a b} \chi_{b}(-1 / \tau)$. For $\tau \rightarrow 0 i, \chi_{b}(-1 / \tau)$ is dominated by the vacuum sector, so that $\chi_{a}(0)=s_{a 1} \chi_{1}(i \infty)[39]$.
} 
The fusion invariance further justifies the probability $p_{a}=d_{a}^{2} / \mathcal{D}^{2}$ as the correct random ensemble. If we consider a random anyon gas in the bulk, and draw two large adjacient regions $A$ and $B$, we expect the anyon type of each region (defined by the fusion of all anyons in that region) to be random, while the same should apply to region $A \cup B$. This is the bulk interpretation why a random distribution $p_{a}$ that emerges from ergodic motion of anyons should be fusion invariant.

With this probability distribution, we can compute the random average of the residue value $r[a, b]$ :

$$
\langle r\rangle:=\sum_{a, b} p_{a} p_{b} r[a, b]=\sum_{a, b} \frac{d_{a}^{2} d_{b}^{2}}{\mathcal{D}^{4}} \frac{\mathcal{D} s_{a b}^{*}}{d_{a} d_{b}}=\sum_{a, b} \frac{s_{1 a} s_{1 b} s_{a b}^{*}}{\mathcal{D}}=\frac{1}{\mathcal{D}^{2}}
$$

In the second last step, we used the unitarity of S-matrix in the summation. Interestingly, the final result only depends on the total quantum dimension $\mathcal{D}$, which also appeared as the characterization of topological entanglement entropy $\gamma=\log \mathcal{D}$, as was proposed in ref. [40, 41]. Therefore we have related a measure of the butterfly effect, the random operator OTOC, with a measure of the topological order, the topological entropy: ${ }^{13}$

$$
\langle r\rangle=e^{-2 \gamma}
$$

We would like to provide some further analysis to this formula. Firstly, we discussed earlier that for fixed channels, scrambling only occurs for non-Abelian channels, since for Abelian channels $a, b,|r[a, b]|=1$. In contrast, for the random operator case, the average $\langle r\rangle<1$ even for an Abelian theory. This is because even in an Abelian theory, nontrivial phase interference can occur between different conformal families for an operator that is a superposition of different families. Physically, even an Abelian fractional statistics requires fractionalization, which in the $(2+1)$-d language means that even an Abelian anyon is a collective excitation of the system which cannot be created by a local operator. Due to such intrinsic nonlocality in the dynamics of the system, the time evolution of a generic operator looks chaotic, although that of a special operator in a single conformal family does not. In this sense, an Abelian theory is an intermediate case between free (boson or fermion) systems and more chaotic (non-Abelian) RCFTs.

Secondly, the total quantum dimension is, roughly speaking, the size of operator content in an RCFT. equation (4.4) means that an RCFT with more fields is on average more chaotic. For an RCFT with $N$ conformal families, $\mathcal{D}^{2}=\sum_{a=1}^{N} d_{a}^{2} \geq N$, such that $\langle r\rangle \leq \frac{1}{N}$. Moreover, the equal sign can only be reached if and only if the theory is Abelian. In other words, for the same number of conformal families (or anyon types in the $(2+1)$-d language), non-Abelian theories are more chaotic than Abelian theories. For example, in the $\mathrm{SU}(2)_{k}$ WZW model, the number of family is $N=k+1$ and the total quantum dimension is $\mathcal{D}=\frac{\sqrt{(k+2) / 2}}{\sin (\pi /(k+2))} \sim k^{3 / 2}$ in large $k$ limit. Therefore, $\langle r\rangle \sim k^{-3}$ in $\mathrm{SU}(2)_{k}$, while in an Abelian theory with the same $N=k+1$ one would have $\langle r\rangle=(k+1)^{-1}$. This is

\footnotetext{
${ }^{13}$ A similar formula appears in ref. [38], where the averaged OTOC is related to the second Renyi entropy of a certain region in the doubled state that represents the time evolution operator. It is possible that these two formula are related, although the relation is not clear to us yet.
} 


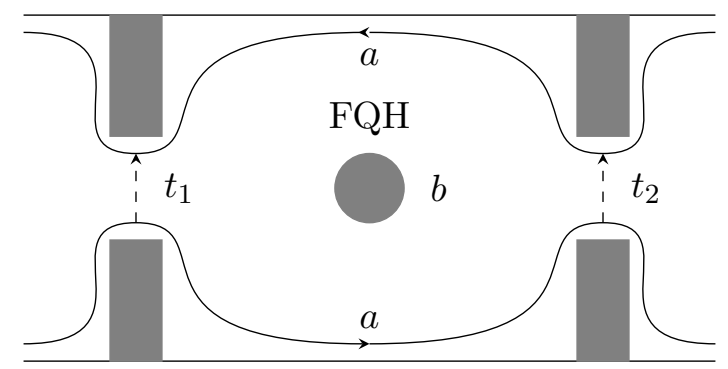

Figure 8. An example of two point-contact interferometry. $t_{1}$ and $t_{2}$ are the tunneling amplitude for left and right "bridges" respectively. The fractional quantum Hall state occupies the region between the two edges (curves with arrow), with the shaded regions depleted. We denote the anyonic charge on the edge and the central island by $a$ and $b$, respectively.

conceptually consistent with our observation in section 2.4 that most channels in $\mathrm{SU}(2)_{k}$ are strongly chaotic in the large $k$ limit.

\section{Conclusion and discussions}

In this article, we studied OTOC in the context of RCFTs, and relate its behavior to the universal algebraic data of RCFT, such as the monodromy matrix and the modular S-matrix. Through the bulk-boundary correspondence of $(2+1)$-d TOS, we pointed out a connection between the OTOC in RCFTs and the fractional statistics in the corresponding TOS. We have shown that the OTOC in an RCFT can be mapped to a time-ordered four-point function which corresponds to a physical process of anyon braiding. In other words, our results point out that the two consequences of "emergent nonlocality" in $(2+1)$-d TOS - chaos on the boundary and fractionalization in the bulk - always accompany each other. Furthermore, our result shows that for a fixed channel (meaning fixed conformal families in the boundary, or fixed anyon types in the bulk), scrambling only occurs for non-Abelian anyons, as a consequence of nontrivial interference between different fusion channels. When we consider a pair of random operators rather than operators in a fixed conformal family, the average value of OTOC is determined by the total quantum dimension, so that the "average degree of chaos" in an RCFT is directly related to the topological entanglement entropy in the bulk.

Besides providing a physical interpretation of the relation between chaos and topological order, the bulk-boundary correspondence we discussed also suggests a potential approach of measuring the OTOC experimentally. In equation (2.7), we show that the residue value $r[a, b]$ of OTOC is determined by the $(1,1)$ element of monodromy matrix $\widetilde{M}[a, b]_{11}$. Interestingly, the same quantity played an essential role in the interferometry of anyons [28, 29], which has been studied extensively in fractional quantum Hall states, both theoretically and experimentally. [42-48]

To be more precise, consider a typical interferometer of FQH state with two point contacts [42], as is shown in figure 8. The physical measurable quantity in this setup is the two terminal conductance (with current flowing from left to right) $\sigma_{x x} \propto\left|t_{1}\right|^{2}+$ $\left|t_{2}\right|^{2}+2 \operatorname{Re}\left(t_{1}^{*} t_{2} e^{i \alpha_{a b}} \widetilde{M}[a, b]_{11}\right)$. The phase factor $\exp \left(i \alpha_{a b}\right)$ includes contributions from the 
Aharonov-Bohm phase and other dynamical phase factors. Therefore we see that the residual value of normalized OTOC $r[a, b]=\widetilde{M}[a, b]_{11}$ plays an essential role in the conductance oscillation. A stronger butterfly effect corresponds to a smaller conductance oscillation. In the extreme case when $\widetilde{M}[a, b]_{11}=0$, no interference can be observed in the conductance. For example such an absence of conductance oscillation has been considered in the filling fraction $\nu=5 / 2$ state as an evidence of non-Abelian statistics [44, 45, 47]. According to our results, the absence of conductance oscillation can also be viewed as a measure of complete scrambling in the corresponding conformal families of the RCFT describing the boundary.

In the end, let us make some more speculations about possible generalizations of our results. Chaos is suppressed by emergent conservation laws which constrains the dynamics of the theory. Therefore it is natural to guess that similar results on OTOC can be obtained in non-critical one-dimensional systems with emergent conservation laws, such as integrable models. The integrability of $(1+1)$-d integrable models is described by the Yang-Baxter equation, which has a similar algebraic structure as that in RCFTs and topological order. Therefore, it is tempting to guess that OTOC in one dimensional integrable models might also capture universal algebraic properties of the model, which we leave for future study.

\section{Acknowledgments}

We would like to acknowledge helpful discussions with Meng Cheng, Tian Lan and Daniel A. Roberts. This work is supported by the National Science Foundation through the grant No. DMR-1151786. Upon finishing this work, we become aware of the parallel work of Pawel Caputa, Tokiro Numasawa and Alvaro Veliz-Osorio [49]. We would like to thank Tokiro Numasawa for informing us of their work before posting their paper.

\section{A Notations and conventions}

In this appendix, we will introduce necessary backgrounds for the notations we used in main text, especially the diagrams. Both subjects of RCFT and anyons have be extensively studied and properly summarized in literature, and we will follow the presentation of lecture note by G. Moore and N. Seiberg [13] and the reference therein for RCFTs, and ref. [30] by A. Kitaev for anyon theories. The purpose of this section is to review the diagrammatic conventions for anyon theories, and explain why we are allowed to use them to describe RCFTs.

Let us start with general RCFTs. Such theories have simple analytic properties in physical correlation functions:

$$
\langle\phi \phi \ldots \phi\rangle \sim \sum_{i, j=1}^{M} g_{i j} \mathcal{F}_{i}(\tau) \overline{\mathcal{F}}_{j}(\bar{\tau})
$$

Where $M$ is a finite number, counting the dimension of the space of conformal blocks. $g_{i j}$ is the coefficient indicating the paring between holomorphic and anti-holomorphic blocks. Holomorphic conformal blocks form a vector space parametrized by moduli $\tau$ (same to anti-holomorphic blocks, by $\bar{\tau}$ ). The moduli describes the shape of a two dimensional manifold, together with the locations of fields inserted. An alternative geometrical 


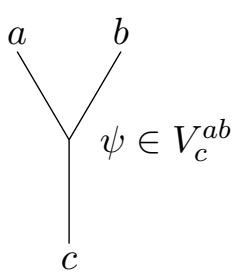

(a) Splitting.

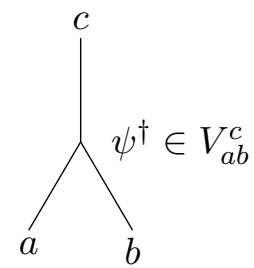

(b) Fusion.

Figure 9. Diagrammatic representation of splitting and fusion space.

formulation due to D. Friedan and S. Shenker [26] describes conformal blocks as a vector bundle over moduli space, and the vector bundle is equipped with a fiber-wise metric $g_{i j}$ for physical correlation functions.

To build a connection to the algebraic theory of anyons, it is essential to find the building blocks on both sides. In RCFT side, such object is the conformal blocks associated to a 3-punctured sphere, or intuitively, the chiral half of three-point functions. ${ }^{14}$ More explicitly, we assume the three punctures were created by insertions of holomorphic fields of family $a, b, c$, and we denote the space of conformal blocks associated to such geometry by $V_{a b c}$. The dimension of this space is known as the fusion multiplicity $N_{a b c}=\operatorname{dim} V_{a b c}$. Such formalism also contains the notion of dual or anti-particles, $\bar{a}, \bar{b}, \bar{c}$. Then we can lift and lower the indices in convention: e.g., $V_{a b c} \simeq V_{c}^{\bar{a} \bar{b}}$. Accordingly, the indices of fusion multiplicity can be lifted or lowered: e.g., $N_{c}^{\bar{a} \bar{b}}=\operatorname{dim} V_{c}^{\bar{a} \bar{b}}=\operatorname{dim} V_{a b c}=N_{a b c}$.

In the algebraic theory of anyons, the parallel notion is the fusion and splitting spaces between "superselection sectors" $a, b$ and $c$ (anyon labels). More concretely, vectors in the splitting space $V_{c}^{a b}$ represent the different ways of splitting $c$ into $a$ and $b$, or equivalent classes of local operators that operate the splitting. Analogously, vectors in the fusion space $V_{a b}^{c}$ represent the different ways of fusing $a$ and $b$ into $c$. If $\psi \in V_{c}^{a b}$, then its dagger $\psi^{\dagger} \in V_{a b}^{c}$, see figure 9. $N_{a b}^{c}=\operatorname{dim} V_{c}^{a b}$ counts the dimension of splitting/fusion space.

On both sides, such objects can be used to build more complicated spaces: in RCFTs, they are conformal blocks of multiple insertions of operators; in anyons, they are fusion/splitting space of multiple anyons. They also have a set of identical consistent conditions to satisfy, of which the crucial one for the fusion theory is the pentagon equation.

Furthermore, both sides have a notion of braiding: in RCFTs the conformal blocks naturally have a dependence of complex coordinates and the braiding of operators is defined. In anyons, braiding of $a$ and $b$ is an element in $V_{a b}^{b a}$ :

$$
R_{a b} \in V_{a b}^{b a}: R_{a b}:=\sum_{a}^{b}
$$

With the braiding we have more consistent conditions to satisfy, known as the hexagon equations.

\footnotetext{
${ }^{14}$ More abstractly and probably more precisely, one can use the notion of chiral vertex operators [32].
} 
In the end the RCFTs naturally requires modular invariance as a physical constraint, which thus leads to the algebraic structure of modular tensor category (MTC). In this paper, we further restrict ourselves to diagonal theories, in which one can choose a proper basis such that the "metric" $g_{i j}=\delta_{i j}$. More explicitly, the physical correlation function has a simpler expression in the diagonal basis:

$$
\langle\phi \phi \ldots \phi\rangle=\sum_{i=1}^{M} \mathcal{F}_{i}(\tau) \overline{\mathcal{F}}_{i}(\bar{\tau})
$$

Under this condition, we end up with unitary modular tensor category (UMC) [27]. On the anyon side, after introducing the braiding, the theory is already physically sensible and known as unitary braided fusion category (UBFC). With extra non-degeneracy condition on braiding, we end up with the same algebraic theory: unitary modular tensor category (UMC). We will restrict ourselves in this paper to discuss those theories described by UMC.

Unfortunately, the literature on RCFTs and anyons have different conventions of drawing diagrams and denoting matrices. We will choose the conventions of anyon theories. More explicitly, we follow the "arrowless" conventions in ref. [30], i.e., the vertical lines should read from bottom to top, and arrows will only be marked on horizontal lines when necessary. For example, in such conventions, the conformal block in equation (2.4) is drawn as a vector in $V_{\bar{a} a \bar{b} b} \simeq V_{b}^{a \bar{a} b}$ :

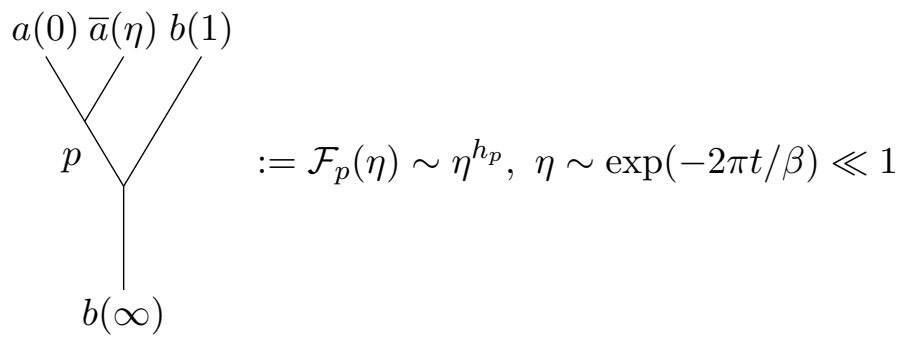

we only write the leading term here for the late time $t \gg \beta$.

\section{B The monodromy matrix $\widetilde{M}[a, b]$}

In this section, we review the algebraic expression of $\widetilde{M}[a, b]$ in terms of F-matrices and R-matrices, and also its diagrammatic expression. Relevant discussions can be found in ref. [50]

Operator $\widetilde{M}$ is a linear map: $V_{b}^{a \bar{a} b} \rightarrow V_{b}^{a \bar{a} b}$.

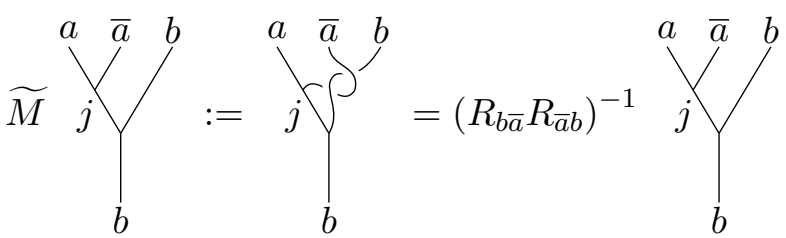


Use bases transformation (F-matrix), we can express $\left(R_{b \bar{a}} R_{\bar{a} b}\right)^{-1}$ operator in terms of $R$ and $F$-matrices.

$$
\begin{aligned}
\left(R_{b \bar{a}} R_{\bar{a} b}\right)^{-1} \sum_{b}^{a} \sum^{\bar{a} b} & =\left(R_{b \bar{a}} R_{\bar{a} b}\right)^{-1} \sum_{k}\left[F_{b}^{a \bar{a} b}\right]_{j k} \sum_{k}^{\bar{a} b} \\
& \left.=\sum_{k}\left[F_{b}^{a \bar{a} b}\right]_{j k}\left(R_{k}^{b \bar{a}} R_{k}^{\bar{a} b}\right)^{-1}\right\}_{k}^{a} b \\
& \left.=\sum_{k, i}\left[F_{b}^{a \bar{a} b}\right]_{j k}\left(R_{k}^{b \bar{a}} R_{k}^{\bar{a} b}\right)^{-1}\left[F_{b}^{a \bar{a} b}\right]_{k i}^{\dagger} \sum^{a}\right\}^{\bar{a}} b
\end{aligned}
$$

In terms of matrix elements:

$$
\widetilde{M}[a, b]_{i j}=\sum_{k}\left[F_{b}^{a \bar{a} b}\right]_{j k}\left(R_{k}^{b \bar{a}} R_{k}^{\bar{a} b}\right)^{-1}\left[F_{b}^{a \bar{a} b}\right]_{k i}^{\dagger}
$$

We should clarify again to avoid confusion that $\widetilde{M}[a, b]_{i j}$ is in general a matrix itself. The space of the conformal block with intermediate channel label " $i$ " (or $j, k)$ in the equation is in general $N_{a \bar{a}}^{i} N_{b \bar{b}}^{i}$ dimensional, which could be greater than 1. Therefore, labels $i, j, k$ should be read as labels for space in general. However, the "monodromy" scalar $\widetilde{M}[a, b]_{11}$ (denoted as $M_{a b}$ in anyon interferometry literature) is indeed a scalar: $N_{a \bar{a}}^{1} N_{b \bar{b}}^{1}=1$ by axioms in both RCFTs [27] and anyons [30].

It is also convenient to rewrite matrix $\left(R_{k}^{b \bar{a}} R_{k}^{\bar{a} b}\right)^{-1}$ in terms of the topological spin $\theta_{a}$, which is related to conventional spin $s_{a}$ by $\theta_{a}=\exp \left(2 \pi i s_{a}\right)$ when the latter is defined. $\left(R_{k}^{b \bar{a}} R_{k}^{\bar{a} b}\right)^{-1}=\frac{\theta_{a} \theta_{b}}{\theta_{k}}$, so that

$$
\widetilde{M}[a, b]_{i j}=\sum_{k}\left[F_{b}^{a \bar{a} b}\right]_{j k}\left(\frac{\theta_{a} \theta_{b}}{\theta_{k}}\right)\left[F_{b}^{a \bar{a} b}\right]_{k i}^{\dagger}
$$

The diagrammatic expression of this equation is easier to memorize. We use the inner product to single out the matrix element $\widetilde{M}[a, b]_{i j}=\frac{\widetilde{\operatorname{Tr}}\left(\psi_{i}^{\dagger} \widetilde{M} \psi_{j}\right)}{\sqrt{\widetilde{\operatorname{Tr}}\left(\psi_{i}^{\dagger} \psi_{i}\right) \widetilde{\operatorname{Tr}}\left(\psi_{j}^{\dagger} \psi_{j}\right)}}$, where denominator (normalization):

$$
\left.\widetilde{\operatorname{Tr}}\left(\psi_{i}^{\dagger} \psi_{i}\right)=a_{i}{ }_{i}^{\dagger}\right\rangle b=d_{a} d_{b}=\widetilde{\operatorname{Tr}}\left(\psi_{j}^{\dagger} \psi_{j}\right)
$$


and numerator:

$$
\left.\widetilde{\operatorname{Tr}}\left(\psi_{i}^{\dagger} \widetilde{M} \psi_{j}\right)=\widetilde{\operatorname{Tr}}\left[\left(Y_{i}^{a} \bar{a}\right)^{\dagger} \cdot \sum_{j}^{a}\right\}^{\bar{a}} b^{b}\right]=b_{j}^{b}
$$

We add back the arrows for horizontal lines to avoid confusion. Therefore, the final diagrammatic representation for element $\widetilde{M}[a, b]_{i j}$ is

$$
\widetilde{M}[a, b]_{i j}=\frac{1}{d_{a} d_{b}}(\overbrace{j}^{i}
$$

In particular, the $(1,1)$ element has a nice expression in terms of the modular S-matrix:

$$
\widetilde{M}[a, b]_{11}=\frac{1}{d_{a} d_{b}} a(b)=\frac{\mathcal{D} s_{a b}^{*}}{d_{a} d_{b}}
$$

More generally, if one introduce the "generalized" S-matrix [27, 30] $S_{z} \in \operatorname{Aut}\left(\mathcal{L}_{z}\right)$, where $\mathcal{L}_{z}=\bigoplus_{b} V_{b}^{b z}$ is the space associated to torus with one puncture of label $z$, the $(1 z)$ or $(z 1)$ element also has simpler algebraic expression in terms of the modular data:

$$
\widetilde{M}[a, b]_{1 z}=\frac{1}{d_{a} d_{b}}\left({ }_{z}=\frac{\mathcal{D} s_{z, a \bar{b}}}{d_{a} d_{b}} ; \quad \widetilde{M}[a, b]_{z 1}=\frac{1}{d_{a} d_{b}}\right.
$$

If we take $z=1, s_{z, a \bar{b}}=s_{\bar{z}, a \bar{b}}=s_{a \bar{b}}=s_{a b}^{*}$ goes back to the familiar S-matrix. (To avoid confusion, we remark here that $s_{z, a b}$ we defined via diagram is different from ref. [30] by a factor of $\sqrt{d_{z}}$ due to the different conventions for diagram normalization. Our $s_{z, a b}$ has normalization: $\sum_{b} s_{z, a b} s_{z, b c}^{*}=d_{z} \delta_{a c}$.)

\section{The residue value $r$ in $\mathrm{SU}(\mathrm{N})_{2}$ WZW models}

In this section, we study the residue value $r$ in SU(N) WZW models at level 2, which are related to the $\mathrm{SU}(2)$ level $N$ models we study in the main text by the level-rank duality.

The explicit formula for modular S-matrix of general $\mathrm{SU}(N)_{k}$ is complicated. However, due to the simplicity of $\mathrm{SU}(2)_{N}$, it is possible to have a simple formula for $\mathrm{SU}(N)_{2}$, which is given by a level-rank duality on S-matrix (for example, see ref. [22]):

$$
s_{\lambda, \mu}=\sqrt{\frac{k}{N}} e^{2 \pi i|\lambda||\mu| / N k} s_{\lambda^{t}, \mu^{t}}^{*}
$$

where reduced Young diagram $\lambda, \mu \in \mathrm{SU}(N)_{k}$, and their transpose $\lambda^{t}, \mu^{t} \in \mathrm{SU}(k)_{N}$, (e.g., see figure 10 as a demonstration). For $k=2$, diagram $\lambda$ can be conveniently parametrized 


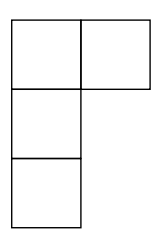

(a)

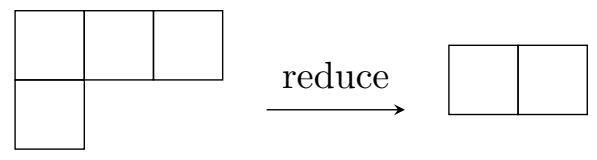

(b)

Figure 10. (a) Example of reduced Young diagram $\lambda$ in $\mathrm{SU}(N)_{2}$ and (b) its transpose $\lambda^{t}$ in $\mathrm{SU}(2)_{N}$. (We assume $N>3$ here). We can label $\lambda$ by $x=3, y=1$ and reduced $\lambda^{t}$ by $x-y=2$.

by two integers: $N-1 \geq x \geq y \geq 0$, which count the number of boxes for column 1 and 2 . Its transpose is not generally reduced in $\mathrm{SU}(2)_{N}$, but reduce to a $\lambda^{t} \in \mathrm{SU}(2)_{N}$, which can be parametrized by one integer $x-y \in[0, N-1] \cap \mathbb{Z}$. And $|\lambda|$ counts the total number of boxes in reduced diagram: $|\lambda|=x+y$. The identity sector corresponds to $x=y=0$. It is also useful to mention the total number of families in $\mathrm{SU}(N)_{k}: \frac{(k+N-1) !}{k !(N-1) !}$.

We can derive the modular S-matrix of $\mathrm{SU}(N)_{2}$ by the explicit formula of the S-matrix of $\mathrm{SU}(2)_{N}$ :

$$
s_{(x, y),\left(x^{\prime}, y^{\prime}\right)}=\frac{2}{\sqrt{N(N+2)}} e^{2 \pi i \frac{(x+y)\left(x^{\prime}+y^{\prime}\right)}{2 N}} \sin \left(\frac{(x-y+1)\left(x^{\prime}-y^{\prime}+1\right)}{N+2} \pi\right)
$$

Here integers $x, y, x^{\prime}, y^{\prime}$ satisfy $N-1 \geq x \geq y \geq 0$ and $N-1 \geq x^{\prime} \geq y^{\prime} \geq 0$. An immediate observation is that when $N \gg 1$, the number of families in $\mathrm{SU}(N)_{2}$ is much greater than those in $\mathrm{SU}(2)_{N}: N(N+1) / 2 \gg N+1$. Therefore, the norm $\left|s_{\lambda, \mu}\right|=\sqrt{\frac{2}{N}}\left|s_{\lambda^{t}, \mu^{t}}\right|$ depends on a smaller set of real numbers $\left|s_{\lambda^{t}, \mu^{t}}\right|$ in $\mathrm{SU}(2)_{N}$.

We can also derive the $|r[\lambda, \mu]|$ of $\mathrm{SU}(N)_{2}$ from the formula C.1:

$$
|r[\lambda, \mu]|=\frac{\left|s_{\lambda^{t}, \mu^{t}}\right|\left|s_{11}\right|}{\left|s_{1, \mu^{t}}\right|\left|s_{1, \lambda^{t}}\right|}=\left|r\left[\lambda^{t}, \mu^{t}\right]\right|
$$

which is identical to those in the dual theory.

In the following we will show explicitly that not only the spectrum of $|r|$ are identical for the dual theories $\mathrm{SU}(N)_{2}$ and $\mathrm{SU}(2)_{N}$, but also the distributions of $|r|$ are identical. $|r[i, j]|$ for two labels $i, j \in \mathrm{SU}(2)_{N}$, i.e. $i, j=0,1,2, \ldots, N$ has the following expression:

$$
|r[i, j]|=\left|\frac{\sin \left(\frac{\pi}{N+2}\right) \sin \left(\frac{(i+1)(j+1) \pi}{N+2}\right)}{\sin \left(\frac{(i+1) \pi}{N+2}\right) \sin \left(\frac{(j+1) \pi}{N+2}\right)}\right|
$$

First of all, it can be directly verified that $|r[i, j]|=|r[i, N-j]|, \forall i, j$, since

$$
\left|\sin \left(\frac{(i+1)(j+1) \pi}{N+2}\right)\right|=\left|\sin \left[\left(1-\frac{(j+1)}{N+2}\right)(i+1) \pi\right]\right|=\left|\sin \left(\frac{(N-j+1)(i+1) \pi}{N+2}\right)\right|
$$

Next, we count how many labels in $\mathrm{SU}(N)_{2}$ are mapped to $j$ and $N-j$ (we assume $j \neq N / 2$ for now, and comment later) in $\mathrm{SU}(2)_{N}$. In general, $j$ in $\mathrm{SU}(2)_{N}$ corresponds to all pairs of integers $(x, y)$ satisfying $x-y=j, N-1 \geq x \geq y \geq 0$. There are 
in total $N-j$ pairs. So together with those corresponds to label $N-j$, there are $N$ channels in $\mathrm{SU}(N)_{2}$ that correspond to the pair of channels $\{j, N-j\}$ in $\mathrm{SU}(2)_{N}$. In other words, for generic two labels $i, j$, there are four identical normed residue values $|r[i, j]|=|r[N-i, j]|=|r[i, N-j]|=|r[N-i, N-j]|$ in $\mathrm{SU}(2)_{N}$, and there are $N^{2}$ identical residual values in $\mathrm{SU}(N)_{2}$. Therefore each $|r[i, j]|$ in $\mathrm{SU}(2)_{N}$ has $N^{2} / 4$ duplicates in $\mathrm{SU}(\mathrm{N})_{2}$. For the case when $j=N-j=N / 2 \in \mathbb{Z}$, there are $N-j=N / 2$ labels in $\mathrm{SU}(N)_{2}$, so that the counting also holds.

The above counting argument is sufficient to prove that the probability distributions for $|r|$ in $\mathrm{SU}(2)_{N}$ and $\mathrm{SU}(N)_{2}$ are identical. We should comment here that we have only discussed the norm of $r . r$ has a strongly fluctuating phase, which can distinguish between different $\lambda^{\prime}$ 's that have same reduced transpose $\lambda^{t} \in \mathrm{SU}(2)_{N}$. This is consistent with the fact that the two dual theories have different average OTOC $\langle r\rangle$ since they have different quantum dimensions.

In parallel with the observation in $\mathrm{SU}(2)_{k}$, where scrambling is more sufficient in larger $k$, we can deduce here that scrambling is more sufficient in large $N$ for $\mathrm{SU}(N)_{2}$ models. Therefore $\mathrm{SU}(N)_{2}$ is an example of a family of RCFTs with strong scrambling in the large central charge limit, which may be interesting from the point of view of holographic duality.

Open Access. This article is distributed under the terms of the Creative Commons Attribution License (CC-BY 4.0), which permits any use, distribution and reproduction in any medium, provided the original author(s) and source are credited.

\section{References}

[1] A. Larkin and Y.N. Ovchinnikov, Quasiclassical method in the theory of superconductivity, Sov. Phys. JETP 28 (1969) 1200.

[2] S.H. Shenker and D. Stanford, Black holes and the butterfly effect, JHEP 03 (2014) 067 [arXiv: 1306.0622] [INSPIRE].

[3] A. Kitaev, Hidden correlations in the Hawking radiation and thermal noise, talk given at Fundamental Physics Prize Symposium, U.S.A. (2014).

[4] J. Maldacena, S.H. Shenker and D. Stanford, A bound on chaos, arXiv:1503.01409 [INSPIRE].

[5] S.H. Shenker and D. Stanford, Multiple shocks, JHEP 12 (2014) 046 [arXiv:1312. 3296] [INSPIRE].

[6] D.A. Roberts, D. Stanford and L. Susskind, Localized shocks, JHEP 03 (2015) 051 [arXiv: 1409.8180] [INSPIRE].

[7] S.H. Shenker and D. Stanford, Stringy effects in scrambling, JHEP 05 (2015) 132 [arXiv: 1412.6087] [INSPIRE].

[8] D.N. Page, Average entropy of a subsystem, Phys. Rev. Lett. 71 (1993) 1291 [gr-qc/9305007] [INSPIRE]

[9] P. Hayden and J. Preskill, Black holes as mirrors: quantum information in random subsystems, JHEP 09 (2007) 120 [arXiv:0708.4025] [INSPIRE].

[10] Y. Sekino and L. Susskind, Fast scramblers, JHEP 10 (2008) 065 [arXiv:0808.2096] [INSPIRE]. 
[11] D. Stanford, Many-body chaos at weak coupling, arXiv:1512.07687 [INSPIRE].

[12] B. Michel, J. Polchinski, V. Rosenhaus and S.J. Suh, Four-point function in the IOP matrix model, JHEP 05 (2016) 048 [arXiv: 1602.06422] [INSPIRE].

[13] G. Moore and N. Seiberg, Lectures on RCFT, Phys. Geom. Topol. (1990) 263 [InSPIRE].

[14] J.M. Leinaas and J. Myrheim, On the theory of identical particles, Nuovo Cim. B 37 (1977) 1 [INSPIRE].

[15] F. Wilczek, Quantum mechanics of fractional spin particles, Phys. Rev. Lett. 49 (1982) 957 [INSPIRE].

[16] D.C. Tsui, H.L. Stormer and A.C. Gossard, Two-dimensional magnetotransport in the extreme quantum limit, Phys. Rev. Lett. 48 (1982) 1559 [INSPIRE].

[17] R.B. Laughlin, Anomalous quantum Hall effect: an incompressible quantum fluid with fractionally charged excitations, Phys. Rev. Lett. 50 (1983) 1395 [INSPIRE].

[18] D.A. Roberts and D. Stanford, Diagnosing chaos using four-point functions in two-dimensional conformal field theory, Phys. Rev. Lett. 115 (2015) 131603 [arXiv: 1412.5123] [INSPIRE].

[19] W. Israel, Thermo field dynamics of black holes, Phys. Lett. A 57 (1976) 107 [INSPIRE].

[20] J.M. Maldacena, Eternal black holes in anti-de Sitter, JHEP 04 (2003) 021 [hep-th/0106112] [INSPIRE].

[21] A.A. Belavin, A.M. Polyakov and A.B. Zamolodchikov, Infinite conformal symmetry in two-dimensional quantum field theory, Nucl. Phys. B 241 (1984) 333 [INSPIRE].

[22] P. Francesco, P. Mathieu and D. Sénéchal, Conformal field theory, Springer Science \& Business Media, Springer Germany (2012).

[23] T. Hartman, Entanglement entropy at large central charge, arXiv:1303.6955 [INSPIRE].

[24] A.L. Fitzpatrick, J. Kaplan and M.T. Walters, Universality of long-distance AdS physics from the CFT bootstrap, JHEP 08 (2014) 145 [arXiv: 1403.6829] [INSPIRE].

[25] C.T. Asplund, A. Bernamonti, F. Galli and T. Hartman, Holographic entanglement entropy from 2d CFT: heavy states and local quenches, JHEP 02 (2015) 171 [arXiv:1410.1392] [INSPIRE].

[26] D. Friedan and S.H. Shenker, The analytic geometry of two-dimensional conformal field theory, Nucl. Phys. B 281 (1987) 509 [InSPIRE].

[27] G.W. Moore and N. Seiberg, Classical and quantum conformal field theory, Commun. Math. Phys. 123 (1989) 177 [INSPIRE].

[28] P. Bonderson, K. Shtengel and J.K. Slingerland, Decoherence of anyonic charge in interferometry measurements, Phys. Rev. Lett. 98 (2007) 070401 [quant-ph/0608119] [INSPIRE].

[29] B.J. Overbosch and F.A. Bais, Inequivalent classes of interference experiments with non-Abelian anyons, Phys. Rev. A 64 (2001) 062107 [quant-ph/0105015] [INSPIRE].

[30] A. Kitaev, Anyons in an exactly solved model and beyond, Annals Phys. 321 (2006) 2 [INSPIRE].

[31] C. Nayak, S.H. Simon, A. Stern, M. Freedman and S. Das Sarma, Non-Abelian anyons and topological quantum computation, Rev. Mod. Phys. 80 (2008) 1083 [INSPIRE]. 
[32] G.W. Moore and N. Seiberg, Polynomial equations for rational conformal field theories, Phys. Lett. B 212 (1988) 451 [INSPIRE].

[33] E. Witten, Non-Abelian bosonization in two-dimensions, Commun. Math. Phys. 92 (1984) 455 [INSPIRE].

[34] V.G. Knizhnik and A.B. Zamolodchikov, Current algebra and Wess-Zumino model in two-dimensions, Nucl. Phys. B 247 (1984) 83 [INSPIRE].

[35] E. Witten, Quantum field theory and the Jones polynomial, Commun. Math. Phys. 121 (1989) 351 [INSPIRE].

[36] G.W. Moore and N. Read, Nonabelions in the fractional quantum Hall effect, Nucl. Phys. B 360 (1991) 362 [INSPIRE].

[37] N. Read and E. Rezayi, Beyond paired quantum Hall states: parafermions and incompressible states in the first excited Landau level, Phys. Rev. B 59 (1999) 8084 [cond-mat/9809384] [INSPIRE].

[38] P. Hosur, X.-L. Qi, D.A. Roberts and B. Yoshida, Chaos in quantum channels, JHEP 02 (2016) 004 [arXiv: 1511.04021] [INSPIRE].

[39] J.L. Cardy, Operator content of two-dimensional conformally invariant theories, Nucl. Phys. B 270 (1986) 186 [INSPIRE].

[40] A. Kitaev and J. Preskill, Topological entanglement entropy, Phys. Rev. Lett. 96 (2006) 110404 [hep-th/0510092] [INSPIRE].

[41] M. Levin and X.-G. Wen, Detecting topological order in a ground state wave function, Phys. Rev. Lett. 96 (2006) 110405 [INSPIRE].

[42] C.d.C. Chamon, D. Freed, S. Kivelson, S. Sondhi and X. Wen, Two point-contact interferometer for quantum Hall systems, Phys. Rev. B 55 (1997) 2331 [cond-mat/9607195].

[43] E.H. Fradkin, C. Nayak, A. Tsvelik and F. Wilczek, A Chern-Simons effective field theory for the Pfaffian quantum Hall state, Nucl. Phys. B 516 (1998) 704 [cond-mat/9711087] [INSPIRE].

[44] P. Bonderson, A. Kitaev and K. Shtengel, Detecting non-Abelian statistics in the $\nu=5 / 2$ fractional quantum Hall state, Phys. Rev. Lett. 96 (2006) 016803 [INSPIRE].

[45] A. Stern and B.I. Halperin, Proposed experiments to probe the non-Abelian $\nu=5 / 2$ quantum Hall state, Phys. Rev. Lett. 96 (2006) 016802 [cond-mat/0508447].

[46] Y. Ji, Y. Chung, D. Sprinzak, M. Heiblum, D. Mahalu and H. Shtrikman, An electronic Mach-Zehnder interferometer, Nature 422 (2003) 415.

[47] R.L. Willett, L.N. Pfeiffer and K. West, Measurement of filling factor 5/2 quasiparticle interference with observation of charge e/4 and e/2 period oscillations, Proc. Nat. Acad. Sci. 106 (2009) 8853 [arXiv:0807.0221].

[48] Y. Zhang et al., Distinct signatures for Coulomb blockade and Aharonov-Bohm interference in electronic Fabry-Perot interferometers, PhysRev. B 79 (2009) 241304 [arXiv:0901.0127].

[49] P. Caputa, T. Numasawa and A. Veliz-Osorio, Scrambling without chaos in RCFT, arXiv: 1602.06542 [INSPIRE].

[50] P.H. Bonderson, Non-Abelian anyons and interferometry, Ph.D. thesis, California Institute of Technology, U.S.A. (2007). 